\title{
Comparison of ACINUS, Caspase-3, and TUNEL as Apoptotic Markers in Determination of Tumor Growth Rates of Clinically Localized Prostate Cancer Using Image Analysis
}

\author{
Swaroop S. Singh ${ }^{\# 1,{ }^{*}, \text { Diana C. Mehedint }}{ }^{\# 2}$, O. Harris Ford III2, D. Antony Jeyaraj ${ }^{2}$, Elena A. \\ Pop $^{2}$, Susan J. Maygarden ${ }^{3}$, Anastasia Ivanova ${ }^{2,4}$, Rameela Chandrasekhar ${ }^{5,6}$, Gregory E. \\ Wilding $^{5,6}$, and James L. Mohler $1,2,7,8$ \\ ${ }^{1}$ Department of Urology, Roswell Park Cancer Institute, Buffalo, New York \\ ${ }^{2}$ Lineberger Comprehensive Cancer Center, The University of North Carolina at Chapel Hill, \\ Chapel Hill, North Carolina \\ ${ }^{3}$ Department of Pathology and Laboratory Medicine, The University of North Carolina at Chapel \\ Hill, Chapel Hill, North Carolina \\ ${ }^{4}$ Department of Biostatistics, The University of North Carolina at Chapel Hill, Chapel Hill, North \\ Carolina \\ ${ }^{5}$ Department of Biostatistics, Roswell Park Cancer Institute, Buffalo, New York \\ ${ }^{6}$ Department of Biostatistics, State University of New York, Buffalo, New York \\ ${ }^{7}$ Department of Surgery, The University of North Carolina at Chapel Hill, Chapel Hill, North \\ Carolina \\ ${ }^{8}$ Department of Urology, State University of New York, Buffalo, New York \\ \# These authors contributed equally to this work.
}

\section{Abstract}

BACKGROUND-The balance between apoptotic and proliferative processes determines the enlargement of a tumor. Accurate measurement of apoptotic and proliferative rates from diagnostic prostate biopsies would allow calculation of tumor growth rates in a population-based prostate cancer $(\mathrm{CaP})$ study. Automated image analysis may be used if proliferation and apoptotic biomarkers provide clearly resolved immunostained images.

METHODS-Clinical CaP aggressiveness was assigned as low, intermediate or high using clinical criteria for 46 research subjects with newly diagnosed CaP. Diagnostic biopsy sections from the research subjects were dual-labeled for proliferation biomarker, Ki-67 and apoptotic biomarker, apoptotic chromatin condensation inducer in the nucleus (ACINUS). Apoptotic biomarkers, caspase- 3 and terminal deoxyribonucleotidyltransferase mediated dUTP-biotin nick end labeling (TUNEL) were labeled separately. Images from immunostained sections were

"Correspondence to: Department of Urology, Roswell Park Cancer Institute, 143 Cell \& Virus Annex, Elm \& Carlton Streets, Buffalo, NY 14263. swaroop.singh@ roswellpark.org. 
analyzed using automated image analysis and tumor growth rates computed. Association between clinical $\mathrm{CaP}$ aggressiveness and tumor growth rates was explored.

RESULTS-Sixteen subjects had high, 17 had intermediate, and 13 had low clinical CaP aggressiveness. Positive immunostaining was localized to the nucleus for Ki-67, ACINUS, and TUNEL. A statistically significant linear trend across clinical CaP aggressiveness categories was found when tumor growth rates were calculated using ACINUS $(P=0.046)$. Logistic regression and ROC plots generated showed ACINUS (AUC $=0.677, P=0.048$ ) and caspase-3 (AUC $=$ $0.694, P=0.038$ ) to be better predictors than TUNEL (AUC $=0.669, P=0.110$ ).

CONCLUSIONS-ACINUS met the criteria for automated image analysis and for calculation of apoptotic rate. Tumor growth rates determined using automated image analysis should be evaluated for clinical prediction of $\mathrm{CaP}$ aggressiveness, treatment response, recurrence, and mortality.

\section{Keywords}

ACINUS; apoptosis; TUNEL; image analysis; tumor growth rate

\section{INTRODUCTION}

Apoptosis is a fundamental and complex biological process that enables an organism to kill and remove unwanted cells during development, normal homeostasis and disease. This active, inherently programmed phenomenon is characterized by nuclear chromatin condensation and oligonucleosomal DNA fragmentation. Apoptosis has been shown to be initiated (or inhibited) by a variety of environmental stimuli, both physiological and pathological, and occurs spontaneously in untreated malignant neoplasms and some types of treatment-induced tumor regression [1].

Spontaneous continuous cell death is an inherent property of malignant neoplasms. Apoptotic and proliferative bodies have been found in large numbers in rapidly growing tumors. The balance between these two processes determines the rate of tumor growth [1]. During 3-7 months of neoadjuvant androgen deprivation therapy (ADT), reduction in prostate cancer $(\mathrm{CaP})$ volume was accompanied by an increase in apoptotic index $(\mathrm{AI})$, the percentage of apoptotic cells in a sample [2]. An increase in apoptotic activity and a decrease in proliferative activity were found after castration in the $\mathrm{LuCaP} 23.1$ human $\mathrm{CaP}$ xenograft model. $\mathrm{CaP}$ recurrence during castration was associated with decreased AI but proliferative activity did not increase [3]. A statistically significant correlation also was found between AI and pathological stage $(P<0.001)$ and Gleason grade $(P<0.001)$. AI independently predicted progression-free probability in men, who underwent radical prostatectomy without adjuvant therapy [4].

$\mathrm{CaP}$ remains the most common malignancy and second leading cause of cancer deaths among men in the United States. African Americans experience a disproportionately high incidence and mortality from CaP compared to Caucasian Americans [5]. The North Carolina-Louisiana Prostate Cancer Project ( $\mathrm{PCaP})$ was developed as a multidisciplinary study to assess social, individual, and tumor-level causes of racial differences in $\mathrm{CaP}$ 
mortality [6]. This population-based study includes incident $\mathrm{CaP}$ cases from more than 1,000 African Americans and 1,000 Caucasian Americans. Tumor growth rate in men with newly diagnosed $\mathrm{CaP}$ will be evaluated to test whether it can be used to predict $\mathrm{CaP}$ recurrence and mortality.

Cellular proliferation and apoptotic rates must be measured accurately in $\mathrm{CaP}$ specimens to calculate tumor growth rate for an individual patient. Cellular proliferation can be determined using Ki-67 or proliferating cell nuclear antigen (PCNA). Both biomarkers are intra-nuclear proteins and therefore produce localized immunostaining appropriate for image analysis. Ki-67 is present during the active phases of the cell cycle (G1, S, G2, and mitosis), but PCNA is not cell-cycle specific [7]. Ki-67 labeling index (Ki-67 LI) has been used to predict $\mathrm{CaP}$ outcome in men after radical prostatectomy [8] and CaP-specific survival in patients managed by watchful waiting [9].

Apoptosis is less conspicuous histologically because it is completed swiftly, leaves no residua, and occurs in topographically scattered distribution [10]. The speed of apoptosis and rapidity of clearance of apoptotic bodies in vivo means that very few apoptotic bodies in a tissue section can represent a considerable degree of cumulative cell loss. Numerically small differences in $\mathrm{AI}$ are therefore of great biological importance [11].

Apoptosis is visualized commonly using terminal deoxyribonucleotidyltransferase (TdT) mediated dUTP-biotin nick end labeling (TUNEL) assay. DNA fragments in situ are detected using TdT to transfer biotin-dUTP to strand breaks of cleaved DNA, which are visualized using immunohistochemical stains [12]. Another method for detecting apoptosis uses the death protease, caspase-3. Caspases are crucial mediators of apoptosis. One major factor involved in the apoptotic oligonucleosomal fragmentation of nuclear DNA is caspase activated DNase (CAD), which in healthy cells exists as a dimer bound to its inhibitor, ICAD. During apoptosis ICAD is cleaved by active caspase-3 leading to release and activation of CAD. Activated CAD cleaves nuclear DNA at the boundaries between nucleosomes and produces oligonucleosomal DNA laddering [13]. Another target protein of caspases during apoptosis is apoptotic chromatin condensation inducer in the nucleus (ACINUS). ACINUS induces chromatin condensation without DNA fragmentation. During apoptosis, ACINUS is cleaved by caspase-3 and an unknown protease to produce a $23-\mathrm{kDa}$ fragment (p23). ACINUS-p23 was found to mediate apoptotic chromatin condensation prior to DNA fragmentation in an in vitro system using permeabilized cells [10]. The expression of caspase-3 and ACINUS in CaP tissue sections can be visualized using immunohistochemical stains.

Morphological characterization by microscopy remains the gold standard for accurate identification of apoptotic and proliferating cells. However, such quantitative measurements are time-consuming and lack objectivity and reproducibility, which makes identification of small changes in large populations difficult. Determination of apoptotic and proliferating indices requires visual examination of 1,000-10,000 cells to identify apoptotic and proliferating cells [14]. For example, commercial software (IDEAS $®)$ ) and a multispectral imaging flow cytometer system was used to detect immunochemically labeled apoptotic cells from large numbers of images of colorectal cancer cell lines [14]. We developed Java- 
based software for automated quantitative nuclear analysis of images from immunostained formalin-fixed, paraffin-embedded prostate tissue sections [15]. This software has characterized successfully the expression of nuclear proteins androgen receptor (AR), Ki-67, and thioredoxin reductase 1 (TrxR1). Development of an automated method for accurate tumor growth rate calculation in prostate tissue sections will allow clinical evaluation of tumor growth rate as a biomarker to predict clinical $\mathrm{CaP}$ aggressiveness and treatment response. The objective of this pilot study was to determine which apoptotic marker is best suited, using automated image analysis, to assess tumor growth rates of newly diagnosed $\mathrm{CaP}$ using diagnostic prostate biopsies from research subjects enrolled in $\mathrm{PCaP}$.

\section{MATERIALS AND METHODS}

\section{Tissue Sections}

Paraffin blocks containing diagnostic prostate biopsy specimens from 46 research subjects were obtained from $\mathrm{PCaP}$. These subjects had no prior treatment for $\mathrm{CaP}$. Four sections of 5 $\mu \mathrm{m}$ thickness were cut from each diagnostic block containing $\mathrm{CaP}$ and 1 block without $\mathrm{CaP}$. One section of each block was stained with hematoxylin and eosin (H\&E) and sent to a referee pathologist for confirmation of diagnosis and Gleason score. All areas of $\mathrm{CaP}$ were encircled. Sections from the first 19 research subjects were stained for each biomarker separately. One section from the remaining 26 subjects was dual stained with Ki-67 and ACINUS and the two adjacent sections were immunostained for TUNEL or caspase-3.

\section{Immunohistochemistry}

Ki-67 and ACINUS-Formalin-fixed, paraffin-embedded diagnostic prostate biopsy sections were deparaffinized, rehydrated through alcohol gradient and antigen-retrieved using Citra buffer (Biocare, Walnut Creek, CA) for $10 \mathrm{~min}$ at $120^{\circ} \mathrm{C}$ and 21 PSI. The cooled sections were incubated with serum block, blocked for endogenous peroxidase using $3 \%$ $\mathrm{H}_{2} \mathrm{O}_{2}$ for $5 \mathrm{~min}$ at $37^{\circ} \mathrm{C}$ and blocked with avidin biotin for $15 \mathrm{~min}$ at $37^{\circ} \mathrm{C}$ (Vector, Burlington, CA). Sections were incubated with anti-Ki-67 (MIB-1 clone, Dako, Carpinteria, CA) primary antibody at $1: 50$ for $2 \mathrm{hr}$ at $37^{\circ} \mathrm{C}$. The sections were amplified using a dextran polymer (EnVision Doublestain System, Dako) and visualized using diaminobenzidine (DAB, Dako). The sections were labeled for ACINUS, using anti-ACINUS primary antibody (ABCAM, Cambridge, MA) at 1:500 for $1 \mathrm{hr}$ at $37^{\circ} \mathrm{C}$, amplified using the dextran polymer (EnVision Doublestain System) and visualized using Fast Red (Dako). Sections were counterstained using hematoxylin and mounted using an aqueous mounting medium.

Caspase-3-Formalin-fixed, paraffin-embedded diagnostic prostate biopsy sections were antigen retrieved and serum blocked as described for Ki-67 and ACINUS. Sections were incubated with anti-caspase-3 (R\&D Systems, Minneapolis, MN) diluted 1:500 for $2 \mathrm{hr}$ at $37^{\circ} \mathrm{C}$. Sections processed without antibody were used as negative controls. Peroxidaselabeled polymer and DAB were used to visualize labeled antibody. Sections were counterstained with hematoxylin, dehydrated with graded concentrations of ethanol and Citrisol V (Fisher Scientific, Pittsburgh, PA) and mounted. 
TUNEL assay-Immunoperoxidase detection using indirect TUNEL method was used to detect apoptosis (In Situ Apoptosis Detection Kit (Chemicon International, Temecula, CA)). Sections were deparaffinized, re-hydrated and washed in PBS. Sections were incubated at room temperature in $20 \mu \mathrm{g} / \mathrm{ml}$ proteinase- $\mathrm{K}$ for $15 \mathrm{~min}$. After rinsing in distilled water, sections were incubated in $3 \% \mathrm{H}_{2} \mathrm{O}_{2}$ for 10 min to block endogenous peroxidase activity. The sections were washed in PBS and TdT reaction enzyme was added for 15-20 min and quenched using stop/wash buffer. The sections were washed three times with PBS. Antidigoxigenin-peroxidase conjugate was added and sections were incubated in a humidified chamber for $30 \mathrm{~min}$ at room temperature. The sections were washed and visualized using DAB. Sections were counterstained with hematoxylin, dehydrated with ethanol and Citrisol V (Fisher Scientific) and mounted.

\section{Image Analysis}

The imaging system consisted of a Leica DMRA2 microscope (Leica Microsystems, Inc., Bannockburn, IL) with a motorized stage controller (Ludl Electronic Products Ltd, Hawthorne, NY) equipped with a Hamamatsu 3CCD digital camera (Hamamatsu Corporation, Bridgewater, NJ) and a FlashPoint 3D image grabber card (Integral Technologies, Indianapolis, IN) in a Pentium 4-based personal computer. Image Pro Plus software (Media Cybernetics, Bethesda, MD) was used to acquire images from areas that corresponded to the encircled $\mathrm{CaP}$. Ten images of 24-bit color depth and spatial resolution of $640 \times 480$ pixels were acquired at $40 \times$ from each section and saved as uncompressed TIFF images. Stromal and necrotic areas and other artifacts were removed from the images. Java-based image analysis software was used to determine the number of stained nuclei in the secretary epithelium [15]. Areas with cytoplasmic immunostaining were assessed visually using Image Pro Plus software (MediaCybernetics). Due to the inability of the software to differentiate clearly between red- and brown-stained nuclei, Ki-67 and ACINUS immunostaining were also visually assessed. The total cell count was determined automatically.

\section{Calculation of Tumor Growth Rate}

The proliferative and apoptotic rates were calculated for each image as:

$$
\text { Prolifera tive Rate }=100 \times\left(\frac{\text { Number of Nuclei Stained Positive for ki }-67}{\text { Total Number of Nuclei Present }}\right)
$$

Apoptotic Rate $=100 \times\left(\frac{\text { Number of Nuclei Stained Positive for ACINUS/Caspase3/TUNEL }}{\text { Total Number of Nuclei Present }}\right)$

Tumor growth rate was computed as:

Tumor growth rate was computed as:

Tumor growth rate $=\log \left(\frac{\text { Proliferation Rate }}{\text { Apoptotic Rate }}\right)$ 


\section{Statistical Analysis}

Measures of tumor growth rates using three apoptotic biomarkers were dichotomized into categories around the median. Logistic regression of clinical $\mathrm{CaP}$ aggressiveness categories on the dichotomized tumor growth rate was performed using the PROC LOGISTIC in SAS software (SAS Institute, Inc., Cary, NC). Tests for linear trend across the ordinal clinical $\mathrm{CaP}$ aggressiveness categories were examined. Additionally, clinical $\mathrm{CaP}$ aggressiveness score was dichotomized into categories representing low versus intermediate or high aggressiveness and logistic regression was then used to model the probability of intermediate/high aggressiveness as a function of the individual biomarkers.

In order to assess the predictive ability of the individual biomarkers, the receiver operating characteristic (ROC) curves for each biomarker were generated using tumor growth rates as predictors for clinical $\mathrm{CaP}$ aggressiveness. The area under each curve (AUC) was computed and compared using the nonparametric approach [16]. A nominal significance level of 0.05 was used in all testing.

\section{RESULTS}

Prostate cancer of $46 \mathrm{PCaP}$ research subjects was classified as high, intermediate or low clinical CaP aggressiveness using clinical criteria [6] (Table I). Sixteen research subjects had high, 17 had intermediate, and 13 had low clinical CaP aggressiveness (Table II).

Immunostaining for apoptosis with TUNEL and ACINUS produced clearly resolved images. Positive immunostaining with caspase- 3 was found in the nucleus and cytoplasm whereas ACINUS and TUNEL immunostaining was localized to the nucleus (Fig. 1). Proliferation marker, Ki-67 produced immunostaining localized to the nucleus. The distribution of tumor growth rates categorized by clinical $\mathrm{CaP}$ aggressiveness showed greater overlap of low and high tumor growth rates for caspase-3 and TUNEL compared to ACINUS (Fig. 2).

Binomial regression of clinical $\mathrm{CaP}$ aggressiveness categories (low, intermediate, high) on dichotomized tumor growth rate using ACINUS $(10<$ Median $\leq 6)$, caspase-3 $(11<$ Median $\leq 5)$ and TUNEL (11 < Median $\leq 5)$ biomarkers provided good discrimination among research subjects with low clinical CaP aggressiveness (Table III). ACINUS also provided good discrimination at high clinical $\mathrm{CaP}$ aggressiveness $(3<$ Median $\leq 10)$. The discrimination of research subjects by each biomarker is illustrated in Figure 3. A statistically significant linear trend was found when tumor growth rate was calculated across clinical CaP aggressiveness categories using ACINUS $(P=0.046)$. When examining the biomarkers individually, results from logistic regression and ROC plots (Fig. 4) indicated ACINUS (AUC $=0.677, P=0.048)$ and capase-3 $(\mathrm{AUC}=0.694, P=0.038)$ had better predictive power for apoptosis than TUNEL (AUC $=0.669, P=0.110$ ). The AUC corresponding to each biomarker did not differ significantly from one another $(P=0.965)$.

\section{DISCUSSION}

Solid tumor growth reflects a balance between cell proliferation and cell death. Tumor growth results from the imbalance of cell gain and cell loss, and quantitative data indicate 
that decreases from cell loss may be the main contributor to tumor growth [17]. Modeling tumor progression using data gathered from individual tumors may also assist in drug development by providing intermediate endpoints [18]. A high ratio of apoptosis to proliferation was found to correlate with improved survival and better prognosis in patients with cervical adenocarcinoma [19] and colorectal cancer [20], while a lower ratio was predictive of shorter survival in patients with glioblastoma multi-forme [21]. A better understanding of the growth rate of $\mathrm{CaP}$ is important not only for evaluating the necessity and type of treatment, but also for determining suitable follow-up intervals.

Apoptosis is important even when present at low frequency in vivo because of its short duration. The technique adopted to quantify apoptosis must be evaluated in the appropriate system being studied. Immunohistochemistry facilitates ready identification of protein biomarkers for rapid, reproducible and accurate assessment of proliferation and apoptosis. In the TUNEL assay, labeling of DNA strands by biotinylated nucleotides and subsequent immunodetection is used to identify cells with strand breakage. However, apoptosis is not the only situation in which sufficient DNA strand breaks occur. For example, necrosis, autolysis and DNA strand breaks that will be repaired subsequently label positively for TUNEL. Under some situations, apoptosis may not result in generation of nucleosomal fragments but in much larger fragments with presumably fewer detectable breaks [22].

Immunostaining for caspase-3 produced nonspecific positive immunostaining. Cytoplasmic stains are difficult to quantify using automated image analysis due the absence of clearly defined cellular boundaries. TUNEL and ACINUS immunostaining produced well resolved images suitable for computer-assisted image analysis. Distribution plots (box plots, Fig. 2) showed that ACINUS showed a marked difference in the distribution of tumor growth rates at low clinical $\mathrm{CaP}$ aggressiveness compared to high aggressiveness. Statistical analysis using binomial regression of clinical $\mathrm{CaP}$ aggressiveness on dichotomized tumor growth rates and AUCs from logistic regression models confirm tumor growth rates using ACINUS better predict clinical $\mathrm{CaP}$ aggressiveness compared to TUNEL. The immunostained images using ACINUS were localized in the nucleus, since the active form for ACINUS is located exclusively in the nucleus.

Java-based software [15] uses color components (hue, saturation, and intensity) of nuclear objects to differentiate immunopositive nuclei from the total population. However, due to the small difference in the levels of hue between the immunostain for Ki-67 (brown) and ACINUS (red), the software could not differentiate the two immunostains. This problem can be rectified by changing the protein used to visualize either of the two immunostains. The software successfully measured the total nuclear count and immuno-positive staining for TUNEL. In the course of the PCaP study, a large number of prostate biopsies will require analysis in order to compare tumor growth rates. Automated image analysis has been shown to quantify the number of nuclei stained in an image in a reliable and reproducible manner. Additional nuclear characteristics, such as mean optical density of nuclear stain and nuclear roundness, obtained from automated image analysis may be used in later studies.

In this study, ACINUS met the criteria for automated image analysis and for calculation of apoptotic rate. Tumor growth rate calculated using ACINUS was a better predictor of 
clinical CaP aggressiveness than when calculated using TUNEL or caspase-3. Tumor growth rate will be evaluated ultimately as a biomarker to predict $\mathrm{CaP}$ aggressiveness, treatment response, recurrence and mortality. Data from the PCaP study will help uncover biological pathways relevant to $\mathrm{CaP}$ aggressiveness, inform the design of screening programs and community-based interventions and aid in development of methods to identify men at high risk of $\mathrm{CaP}$ mortality.

\section{ACKNOWLEDGMENTS}

PCaP is supported by the Department of Defense Prostate Cancer Research Program (DAMD 17-03-2-0052). The authors thank the staff, advisory committees and research subjects participating in the PCaP study for their significant contributions and Michael Ray, Roswell Park Cancer Institute, for assistance in preparing this manuscript. This study was supported by the NCI Cancer Center Support Grant to Roswell Park Cancer Institute (CA016156) and Lineberger Comprehensive Cancer Center (CA34026).

Grant sponsor: Department of Defense; Grant number: 17-03-2-0052; Grant sponsor: Roswell Park Cancer Institute; Grant number: CA016156; Grant sponsor: Lineberger Comprehensive Cancer Center; Grant number: CA34026.

\section{REFERENCES}

1. Kerr JF, Wyllie AH, Currie AR. Apoptosis: A basic biological phenomenon with wide-ranging implications in tissue kinetics. Br J Cancer. 1972; 26(4):239-257. [PubMed: 4561027]

2. Miyata Y, Kanda S, Sakai H, Hakariya T, Kanetake H. Relationship between changes in prostate cancer cell proliferation, apoptotic index, and expression of apoptosis-related proteins by neoadjuvant hormonal therapy and duration of such treatment. Urology. 2005; 65(6):1238-1243. [PubMed: 15922418]

3. Bladou F, Vessella RL, Buhler KR, Ellis WJ, True LD, Lange PH. Cell proliferation and apoptosis during prostatic tumor xeno-graft involution and regrowth after castration. Int J Cancer. 1996; 67:785-790. [PubMed: 8824549]

4. Dachille G, Cai T, Ludovico GM, Vestita G, Pagliarulo G, Nesi G, Mazzoli S, Meacci F, Mondaini N, Detti B, Bartoletti R. Prognostic role of cell apoptotic rate in prostate cancer: Outcome of a longtime follow-up study. Oncol Rep. 2008; 19(2):541-545. [PubMed: 18202805]

5. Jemal A, Siegel R, Ward E, Hao Y, Xu J, Murray T, Thun MJ. Cancer statistics. CA Cancer J Clin. 2008; 58:71-96. [PubMed: 18287387]

6. Schroeder JC, Bensen JT, Su LJ, Mishel M, Ivanova A, Smith GJ, Godley PA, Fontham ET, Mohler JL. The North Carolina-Louisiana Prostate Cancer Project (PCaP): Methods and design of a multidisciplinary population-based cohort study of racial differences in prostate cancer outcomes. Prostate. 2006; 66(11):1162-1176. [PubMed: 16676364]

7. Gerdes J, Lemke H, Baisch H, Wacker HH, Schwab U, Stein H. Cell cycle analysis of a cell proliferation-associated human nuclear antigen defined by the monoclonal antibody Ki-67. J Immunol. 1984; 133:1710-1715. [PubMed: 6206131]

8. Bettencourt MC, Bauer JJ, Sesterhenn IA, Mostofi FK, McLeod DG, Moul JW. Ki-67 expression is a prognostic marker of prostate cancer recurrence after radical prostatectomy. J Urol. 1996; 156(3): 1064-1068. [PubMed: 8709308]

9. Stattin P, Damber JE, Karlberg L, Bergh A. Cell proliferation assessed by Ki-67 immunoreactivity on formalin fixed tissues is a predictive factor for survival in prostate cancer. J Urol. 1997; 157(1): 219-222. [PubMed: 8976256]

10. Sahara S, Aoto M, Eguchi Y, Imamoto N, Yoneda Y, Tsujimoto Y. ACINUS is a caspase-3activated protein required for apoptotic chromatin condensation. Nature. 1999; 401(6749):168173. [PubMed: 10490026]

11. Bellamy CO, Malcomson RD, Harrison DJ, Wyllie AH. Cell death in health and disease: The biology and regulation of apoptosis. Semin Cancer Biol. 1995; 6(1):3-16. [PubMed: 7548839] 
12. Gavrieli Y, Sherman Y, Ben-Sasson SA. Identification of programmed cell death in situ via specific labeling of nuclear DNA fragmentation. J Cell Biol. 1992; 119(3):493-501. [PubMed: 1400587]

13. Enari M, Sakahira H, Yokoyama H, Okawa K, Iwamatsu A, Nagata S. A caspase-activated DNase that degrades DNA during apoptosis, and its inhibitor ICAD. Nature. 1998; 391(6662):43-50. [PubMed: 9422506]

14. Henery S, George T, Hall B, Basiji D, Ortyn W, Morrissey P. Quantitative image based apoptotic index measurement using multispectral imaging flow cytometry: A comparison with standard photometric methods. Apoptosis. 2008; 13(8):1054-1063. [PubMed: 18543109]

15. Singh SS, Kim D, Mohler JL. Java Web Start based software for automated quantitative nuclear analysis of prostate cancer and benign prostate hyperplasia. Biomed Eng Online. 2005; 4:31. [PubMed: 15888205]

16. DeLong ER, DeLong DM, Clarke-Pearson DL. Comparing the areas under two or more correlated receiver operating character istic curves: A nonparametric approach. Biometrics. 1988; 44(3):837845. [PubMed: 3203132]

17. Arends MJ, McGregor AH, Wyllie AH. Apoptosis is inversely related to necrosis and determines net growth in tumors bearing constitutively expressed myc, ras, and HPV oncogenes. Am J Pathol. 1994; 144(5):1045-1057. [PubMed: 8178928]

18. Stein WD, Figg WD, Dahut W, Stein AD, Hoshen MB, Price D, Bates SE, Fojo T. Tumor growth rates derived from data for patients in a clinical trial correlate strongly with patient survival: A novel strategy for evaluation of clinical trial data. Oncologist. 2008; 13(10):1046-1054. [PubMed: 18838440]

19. Sheridan MT, Cooper RA, West CM. A high ratio of apoptosis to proliferation correlates with improved survival after radiotherapy for cervical adenocarcinoma. Int J Radiat Oncol Biol Phys. 1999; 44:507-512. [PubMed: 10348278]

20. Koike M. Significance of spontaneous apoptosis during color-ectal tumorigenesis. J Surg Oncol. 1996; 62(2):97-108. [PubMed: 8649048]

21. Kuriyama H, Lamborn KR, O'Fallon JR, Iturria N, Sebo T, Schaefer PL, Scheithauer BW, Buckner JC, Kuriyama N, Jenkins RB, Israel MA. Prognostic significance of an apoptotic index and apoptosis/proliferation ratio for patients with high-grade astrocytomas. Neuro Oncol. 2002; 4(3):179-186. [PubMed: 12084348]

22. Harrison DJ. Counting apoptosis-Why and how? J Clin Path Mol Pathol. 1996; 49:M245-M246. 


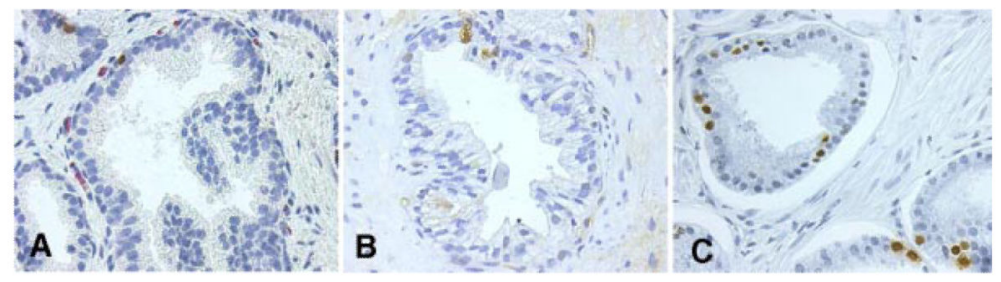

Fig. 1.

A: Ki-67 (brown) and ACINUS (red), (B) caspase-3 (brown), and (C) TUNEL (brown) immunostained images. 


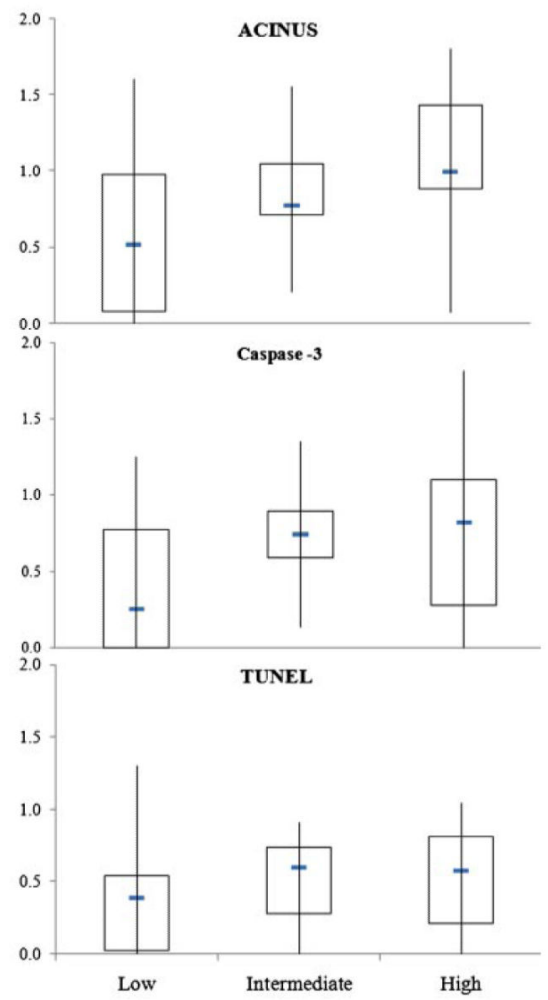

Fig. 2.

Distribution of tumor growth rate measurements using 3 apoptotic biomarkers in 46 research subjects categorized by clinical $\mathrm{CaP}$ aggressiveness. 


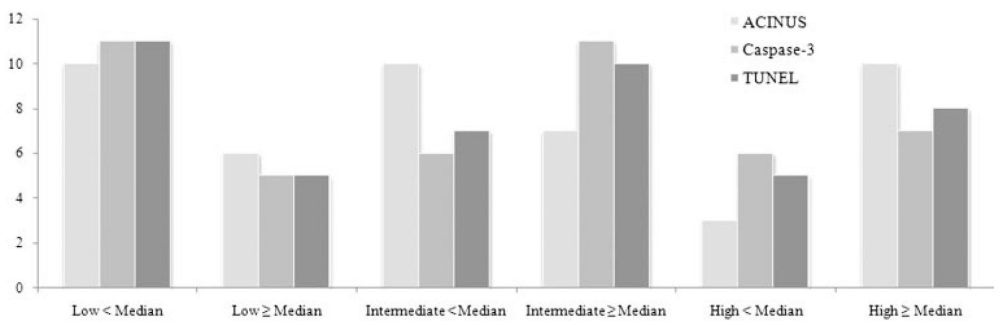

Fig. 3.

Number of research subjects with low, intermediate and high clinical CaP aggressiveness and tumor growth rates above or below the median. 


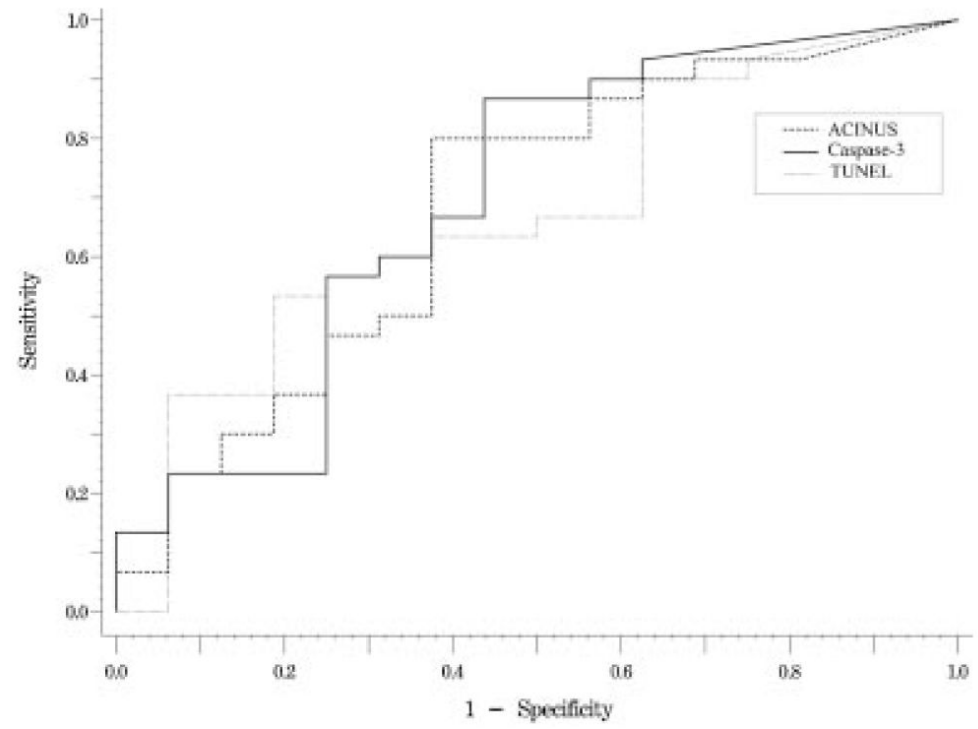

Fig. 4.

ROC curves generated using tumor growth rates from ACINUS, caspase-3 and TUNEL as predictors of clinical $\mathrm{CaP}$ aggressiveness. 
TABLE I

Assignment of Clinical CaP Aggressiveness Using Clinical Criteria

\begin{tabular}{ll}
\hline $\begin{array}{l}\text { Clinical CaP } \\
\text { aggressiveness }\end{array}$ & Clinical criteria \\
\hline Low & $\begin{array}{l}\text { Gleason sum }<7 \text { and } \\
\text { Clinical stage T1-T2 and } \\
\text { PSA }<10\end{array}$ \\
High & Gleason sum $\geq 8$ or \\
& PSA $>20$ or \\
& Gleason sum $=7$ and \\
& Clinical stage T3-T4 \\
Intermediate & All other research subjects \\
\hline
\end{tabular}

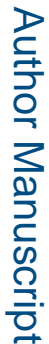

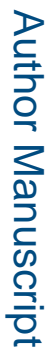


TABLE II

Clinical Characteristics of 46 Research Subjects

\begin{tabular}{lr}
\hline Clinical characteristics & $\begin{array}{r}\text { Number of } \\
\text { research subjects }\end{array}$ \\
\hline Race & 23 \\
African American & 23 \\
Caucasian American & \\
Serum PSA level & 33 \\
$<10$ & 9 \\
$\geq 10$ and $<20$ & 4 \\
220 & \\
Clinical stage & 27 \\
T1 & 18 \\
T2 & 1 \\
T3 & \\
Gleason sum & 20 \\
$<7$ & 17 \\
$=7$ & 9 \\
28 & 16 \\
Clinical CaP aggressiveness & \\
Low & 13 \\
Intermediate & \\
High & \\
\hline
\end{tabular}




\section{TABLE III}

Research Subjects Categorized Into Low, Intermediate and High Clinical CaP Aggressiveness and Dichotomized About the Median of Tumor Growth Rate Measurements

\begin{tabular}{lrrrr}
\hline $\begin{array}{l}\text { Tumor } \\
\text { growth rate } \\
\text { calculated } \\
\text { using }\end{array}$ & \begin{tabular}{r} 
Low \\
\cline { 2 - 5 }$(\mathbf{1 6})$
\end{tabular} & $\begin{array}{r}\text { Intermediate } \\
(\mathbf{1 7})\end{array}$ & $\begin{array}{r}\text { High } \\
(\mathbf{1 3})\end{array}$ & $\begin{array}{r}\text { Trend, } \\
\boldsymbol{P} \text {-value }\end{array}$ \\
\hline ACINUS & & & & \\
$\quad<$ Median & 10 & 10 & 3 & 0.046 \\
$\quad$ MMedian & 6 & 7 & 10 & \\
Caspase-3 & & & & \\
$\quad$ <Median & 11 & 6 & 6 & 0.197 \\
$\quad$ घMedian & 5 & 11 & 7 & \\
TUNEL & & & & \\
$\quad$ <Median & 11 & 7 & 5 & 0.099 \\
$\quad$ MMedian & 5 & 10 & 8 & \\
\hline
\end{tabular}

$P$-value indicate calculated trend at $95 \%$ confidence interval. 